\title{
Surveillance \& Society \\ Intelligent Personal Assistants and the Article Intercultural Negotiations of Dataveillance in Platformed Households
}

\section{Jason Pridmore}

Erasmus University, The Netherlands

pridmore@eshcc.eur.nl

\section{Jessica Vitak}

University of Maryland, USA

jvitak@umd.edu

\section{Daniel Trottier}

Erasmus University, The Netherlands trottier@eshcc.eur.nl

\section{Yuting Liao}

University of Maryland, USA

yliao598@umd.edu

\section{Michael Zimmer}

University of Wisconsin, USA

zimmerm@uwm.edu

\section{Anouk Mols}

Erasmus University, The Netherlands mols@eshcc.eur.nl

\section{Priya C. Kumar}

University of Maryland, USA

pkumar12@umd.edu

\begin{abstract}
The platformization of households is increasingly possible with the introduction of "intelligent personal assistants" (IPAs) embedded in smart, always-listening speakers and screens, such as Google Home and the Amazon Echo. These devices exemplify Zuboff's "surveillance capitalism" by commodifying familial and social spaces and funneling data into corporate networks. However, the motivations driving the development of these platforms - and the dataveillance they afford—vary: Amazon appears focused on collecting user data to drive personalized sales across its shopping platform, while Google relies on its vast dataveillance infrastructure to build its AI-driven targeted advertising platform. This paper draws on cross-cultural focus groups regarding IPAs in the Netherlands and the United States. It reveals how respondents in these two countries articulate divergent ways of negotiating the dataveillance affordances and privacy concerns of these IPA platforms. These findings suggest the need for a nuanced approach to combating and limiting the potential harms of these home devices, which may otherwise be seen as equivalents.
\end{abstract}

\section{Introduction}

On October 24, 2018, Google Home devices became available to consumers in the Netherlands, Sweden, Norway, and Denmark to be used in their native languages. This marks the first time a major platform 
released a voice-activated "Intelligent Personal Assistant" (IPA) for the home in these countries and reflects a push by Google to become increasingly present throughout Europe. This comes two years after Amazon and Google launched devices in the UK and Germany and four years after Amazon's Echo first became available in the United States.

Google's and Amazon's decision to compete in European markets and invest in languages beyond English reflects an increasing "platformization" of the home through the Internet of Things (IoT) and other cloudcomputing technologies. This concept of platformization has been described as the "rise of the platform as the dominant infrastructural and economic model of the social web" (Helmond 2015: 1). The rollout of IPAs to non-English-speaking countries has begun in earnest, with a number of platforms like Apple attempting to similarly penetrate markets and making strategic alliances to ensure their technology is connected within households. ${ }^{1}$ IPAs' smart, always-listening speakers and screens exemplify the idea of "surveillance capitalism" (Zuboff 2019) by commodifying familial and social spaces and funneling relevant data from these locations into corporate networks. While it is unclear how widespread or lasting the success of these devices may be, their growth in the United States - and now in Europe - raises significant privacy and security concerns related to the presence of these platforms in previously private spaces.

In the home and beyond, the motivations driving the development of these devices for platforms - and the dataveillance they afford - vary: Amazon appears focused on collecting user data to drive personalized sales across its shopping platform, while Google relies on its vast dataveillance infrastructure to build its AIdriven targeted advertising platform. This dataveillance (Clarke 1988), or data surveillance, refers to the ongoing collection and exchange of information about individuals facilitated by advanced digital information technologies and computer databases. To better understand the implications and user concerns about security, privacy, and (data) surveillance through these devices, this paper draws on a cross-cultural study of attitudes toward IPAs in the Netherlands and the United States. Through an analysis of 17 focus groups across the two countries, we explore how users and non-users of home IPAs understand, negotiate, and respond to the platforms that support these devices. Findings highlight how users and non-users articulate strategies for negotiating the dataveillance affordances and privacy concerns of IPA platforms. While many Americans in our study either described a sense of resignation toward surveillance technologies or focused on the technologies' benefits over privacy concerns, Dutch participants exhibited much greater wariness toward these platforms' data collection. The findings further suggest the need for a nuanced approach to combating and limiting the potential harms of these home devices and their associated platforms, with a focus on raising critical questions before device use is fully normalized.

\section{Context and Methods}

The integration of platform-based IPAs in home environments has made certain concerns about privacy issues and data collection more ubiquitous (Zeng, Mare, and Roesner 2017). Recent events - including Amazon's Echo unintentional recording of a private conversation and sending it to one of the owner's contacts (Sacks 2018) as well as unprompted laughter from Echo devices (Zeng, Mare, and Roesner 2017) have brought these concerns to the foreground. However, with more than 50 million predicted shipments in 2018 and expected market growth of US\$56.3 million worldwide, these voice-activated IPAs are currently the fastest-growing consumer technology (Perez 2018). Within the US, 15.4\% of the population owns an Amazon Echo and 7.7\% owns a Google Home, while in the UK, the total number of IPA owners has doubled from $5 \%$ to $10 \%$ in one year (Feldman 2018). In Germany, 5.9\% of the population owns an Amazon device and $1.2 \%$ owns a Google Home (Brandt 2018). It will be several years before we get a clear sense of the global proliferation of these devices.

IPAs in home environments seek to simplify performing everyday tasks - such as those related to ecommerce, web search, music streaming, and the control of smart home devices-by allowing users to control the device through speaking (Perez 2018). All of this is (supposed to be) seamlessly integrated

\footnotetext{
${ }^{1}$ Microsoft, for instance, has focused on integrating its IPA Cortana with Amazon's Alexa (see Warren 2018).
} 
through one device, with two platforms - Amazon and Google - most successfully competing in this space. The goals for both companies differ, with Google attempting to capture "micro moments" or intent-driven moments of decision making and preference that happen in the home (Ramaswamy 2015) and Amazon aiming to increase purchase behavior within the platform. However, despite immense market growth and increasing influence of personal assistants on everyday life, actual user behavior is still largely unexplored. While a new era of technology through voice computing is on the rise, research about the integration and the experiences of IPAs is mainly unexplored (Porcheron, Fischer, and Sharples 2017).

Taking the perspective that users matter in the development and implications of new technology (see Oudshoorn and Pinch 2003), this study is based on data collected within focus groups conducted in the US and the Netherlands. Participants were recruited from a survey sample of 9000 university staff (3000 per university) at three universities - two in in the US $(n=1160)$ and one in the Netherlands $(n=325)$. The survey included questions about smartphone use frequency, general privacy and mobile concerns, smartphone data sharing, perceptions and the use of intelligent personal assistants (IPAs) on phones, and perceptions of home IPA devices. It was also used to recruit participants for follow-up focus groups, which are the focus of the current study's analysis. In total, 17 focus groups were conducted, six in the Netherlands $(n=36)$ and 11 in the US ( $n=65)$. A thematic analysis (see Guest, MacQueen, and Namey 2011; Braun and Clarke 2006) of transcripts identified the perceived benefits and detriments of home devices as well as shared concerns among US and Dutch participants. As noted below, there are differences in experiences with these devices given their presence in the US market and absence in the Netherlands. While such unfamiliarity added to Dutch participants' concerns about the devices, their prior understandings and use of IPAs on mobile phones coupled with hands-on experience with one such device during the focus group informed their responses. Our presentation of findings uses pseudonyms to protect participants' identities. Below, we highlight prominent themes that emerged in the US and Netherlands and compare cultural differences between users and non-users in the two countries.

\section{Consumer Desire for Household IPAS—and the Convenience They Bring}

People concerned about increasing surveillance in their everyday lives may be skeptical of buying a home device that constantly listens for trigger words. That said, both "early adopters" and regular users of IPAs see (significant) benefits in using these devices. As noted by Lau, Zimmerman, and Schaub (2018: 9), convenience was a key motivator for IPA use alongside a desire to be seen as an early adopter. In the current study — where data were collected during the first half of 2018 - there was a difference in availability of IPAs for purchase (with devices not yet available in the Netherlands); however, participants' responses across contexts resonated with other research that notes advantages of these devices. Household IPAs were seen as (potentially) beneficial to participants' everyday lives for reasons of hands-free accessibility, ease of use, and their informative and entertaining qualities.

Given the dramatically higher levels of awareness of and experience with IPAs by US participants, we observed some distinct differences toward adopting these devices in the home. A number of US users were very enthusiastic — or described other people in their homes who were enthusiastic - about the use of a voice activated IPA. Mary, for instance, said her husband exemplified the early-adopter mindset: "My husband is obsessed with it so we use it. . . It controls lights in our house and different technologies. We put our shopping list on it so we could just say it out loud. If we're cooking or something, then we throw it on there. We play a lot of music with it as well." Similarly, the benefits of using a voice-activated device were noted by Suchi: "an advantage would be the hands free-ness. It's more like having someone there to dictate to and say, 'I need to have this done,' versus putting in the effort of typing in what it is that you want to do and have that take out time." In line with prior research (Lopatovska et al. forthcoming; Luger and Sellen 2016) that notes the frequent use of these devices for information-related searches (e.g., weather information), entertainment (e.g., playing music) or controlling external devices (e.g., turning lights on and off), other US users we spoke to also highlighted these practices. The emphasis on the advantages of having an IPA in the home was perhaps best described by Julie who made clear that these devices "work in a way that makes my life easier and makes me more efficient ... opens up time for me to do or explore other things.... I like the 
idea of being able to have technology to be able to make me more efficient or my life more efficient in some way."

Although most Dutch participants had never used a home IPA before - and were introduced to the device through brief interactions with one during the focus group - they related some of the devices' convenience features to their use of other technologies, such as phone-based IPAs and other "smart" devices. Peggy described how IPAs could provide a useful solution in her house, where "the location of our light switch is a bit inconvenient. So, you just open the door and say that the lights need to be switched on, small practical things like that, ... just the little things that make life a bit better." Similar to the US participants, those in the Netherlands saw the idea that IPAs help with little things as a key benefit. Claire noted the possibilities of these devices for everyday tasks such as cooking: "I discovered that you can set multiple timers, so . . . when you want to boil potatoes, you set a timer. And eh, you have something in the oven, you set a second timer. And you can set as many timers as you want." While these examples indicate some limited experience among the Dutch participants, they reiterate some of the appeal noted elsewhere of routinizing everyday practices - sometimes well and sometimes not so well (see experiences described in Luger and Sellen 2016).

\section{Consumers' Concerns about IPAS}

While participants identified a number of clear benefits, they also articulated a complex set of concerns about the use or potential use of these devices. While these primarily focused on the technology itself and the risks associated with it, our participants also described ambivalence toward the devices' platforms. The complexities of concerns with household IPAs were often blurred; however, three themes emerged: an over-reliance on technology shifting into forms of technological control, the security risks in technology knowing your routines, and fears surrounding vulnerabilities in the technology in the form of breaches or hacks.

Jennifer, a Dutch participant, was particularly concerned about how IPAs would affect human behavior: "I really wonder why on earth they want you to command something from your bed: 'Make me coffee' and [that suggests] that it really takes too much effort to go to your coffee machine to make coffee. That seems unhealthy to me; it makes you that lazy." This concern was echoed by American participant Eric, whose "biggest worry is that as we use technology more, we get lazier. Like in 20 years, people are just fatter and their brain is smaller, probably." Leah, a Dutch participant with no prior knowledge about household IPAs, was concerned after watching a commercial about Google Home. She feared that "you don't have to know anything anymore. That Google does everything for you and knows about your flight and your [restaurant] reservations. That you won't remember this, and you just ask." This orientation to potential technological omnipotence was also reflected in a number of US participants' comments. Steven was aware of the potential control he was relinquishing to the platform and set limits on his IPA. However, he also described a somewhat fatalistic attitude when he said, "I know it's there. I know it's present. I assume it's listening. We play music. We ask for the time. I keep it simple, it's not attached to anything in my house that could change my environment, lock it, anything. It's fun and convenient, and I try to keep it that so I can preserve some of my privacy, but I recognize some of it's just out of my control." This idea of control was a significant concern across both the US and Dutch contexts, reiterating findings highlighted by Lau et. al. (2018) that despite some built-in protections, users and non-users perceived these devices to have unmet privacy control needs.

IPAs are designed to learn from users" everyday routines and behaviors and integrate into the "smart home" environment. This concerned some participants. In the Netherlands, Andreas had some clear concerns about this potential as he noted, "The moment when you put this thing in your house, it knows when you'll ask: Turn off the lights. You will always do that around the same time at night. So implicitly, a lot of your behavior is measured, even if it is not the in room where you are present at that moment." Likewise, Peggy, another Dutch participant, focused more on other people accessing this information: "You're kind of being eavesdropped [on] permanently with [these devices]. I think, okay, what happens with those data? Because if people would hack it, ... they can easily see when you're not at home, when there are no activities, and 
when you would look at [potential] break-ins." These sorts of concerns came up in the US focus groups as well but often focused on physical security rather than concerns about devices "listening in." American participant Pamela said she was less concerned about the data than the potential for her home to be broken into: "The only concern I have . . is smart locks. If you hook it up, a hacker could, kind of, get into your Alexa and unlock your home that way. We've chosen not to get [smart locks in] our home. We are trying to slowly buy things to make it a smart home. But that's one aspect that we are not going to pursue because of that privacy."

\section{Resignation to Surveillance and Platform Profiling}

The convenience of the IPA devices and the notion that users have "nothing to hide" was prominent in the US focus groups, marking a key divergence in perspectives among American and Dutch participants. Within the US focus groups, many participants felt their mundane activities were of little interest should these devices be used for surveillance purposes. According to Kevin, "I have little concern, whether it's Alexa listening to me or the NSA monitoring my phone calls. . . . I hope they're amused "cause I got nothing to hide. I really don't." Susan saw being continually monitored as a risk but accepted it because of her own perception of her life: "I feel like it's a possible risk that you have when you have these devices. And you either accept that it's a possible risk or you don't. . . I feel like my life is too mundane to worry about it." This orientation was reiterated by several American users, like Jeff: "I don't think anybody else is interested in my life;" and George: "if you're gonna be that concerned about a device listening in, it's because chances are you're probably doing something that you really don't want people overhearing;" and Sam: "my data is already out there, so at this point, I don't have this much of an attachment to protecting the data or being concerned about where my data is going." This belief in a unremarkable life, free from problematic social choices in which personal data is either already out there or seen as uninteresting, pervades studies of surveillance attitudes (see, for instance, Zimmer et al. forthcoming; Mols and Janssen 2017; Solove 2011). Yet, the orientation to platforms that emerged in our research highlighted some differences within the focus groups.

Alongside the idea that participants had uninteresting lives and nothing to hide, there was a general acceptance amongst American participants that profiling practices were an inevitable part of using IPAs. Kevin, in talking about Amazon's Alexa, said it clearly: "I think marketers use it to make a customer profile, the types of things that you might be interested in or are interested in. . . Again, none of that worries me because there's nothing that I would share that Alexa would hear that would embarrass me at any point in time." Likewise, Barbara was "fine" with "information being used for marketing." This acceptance of marketing also aligned with Sam's orientation to Google: "that you, like, opt in with the idea with using a lot of Google products . . . they sort of, like, try to use your data to optimize your experience. So, you sort of opt in to that knowing that they're already going to be looking at it, and so I'm cool with it."

Notably, while many American participants accepted surveillance because of the benefits they received from it, other US participants described a more ambivalent attitude toward platforms when it came to security issues, particularly with sensitive data or government use of their data. This was expressed most succinctly by Mai: "We're trusting Google that what they show me I can listen to is what they kept. For the most part, I trust Google on that, and Amazon. But there's that open concern . . . what are you opening yourself up to? One, that's a government request. And how friendly they are with them. I'm not afraid of that, but just for a principled point of view, where that might lead us?" Likewise, Mike described serious concerns about sensitive data being shared with platforms: "The only time that I really am critical about what I'm talking about is if I'm going to do a credit card transaction or social security number, I will actually go physically mute or even unplug at times."

Dutch participants were far more wary of platforms' intentions than their American counterparts. After discussing Google Home, Alex noted that "Amazon also has such a [device]. They obviously want you to order at Amazon and that [orientation] will be well developed within that hardware." Robert also expressed concerns about large platforms that collected and aggregated user data across sites and shared data with 
third parties. He said, "If you look at what Google does, yes, that links to other parties. Just like Facebook selling their data, Google does exactly the same thing. And that worries me. It is not that you're in contact with Google, but what will that third party do with your data? And how anonymous is your data?" Interestingly, Apple - which is a much smaller player than Google and Amazon in this market - was viewed more positively, particularly among Dutch participants, with Robert commenting that Apple is focused on selling hardware rather than user data.

\section{Discussion}

The arrival of voice-activated IPAs for household use represents a further colonization of platforms into everyday life. To date, it is a limited one, with only a few major players. Despite efforts to create more opensource voice-activated IPAs such as Mycroft AI (https://mycroft.ai/), these devices are made possible by and tethered to particular large-scale platform investments. In the home, their connection to other IoT devices and cloud-based services becomes part of the data-gathering process that creates new avenues for understanding consumer behavior and knowledge for platform owners to either sell for advertising (Google) or engage in increasing platform specific consumption (Amazon). In both cases, however, household IPA devices serve to increase the data-gathering capacities of platforms into the very intimate sphere of people's homes.

Despite this, our study into the expectations and lived experiences of (would be) household IPA users shows fragmentation and inconsistencies in responding to and using these devices. There are clear opportunities and utility seen in the devices, mixed with both concern and apathy about the data they produce and the risks they may pose. However, the differences between expectations in the two locations of our research highlight both cultural and experiential differences. Among Dutch participants, particularly those with limited exposure to household IPAs, there is more concern about the platforms and what they will do with data gained by these devices than among their US counterparts.

If we take seriously the notion that users can and do shape the use and development of technology and the policies surrounding them (Oudshoorn and Pinch 2003), we are at an opportune moment, given current trajectories, to call into question the continued datafication of household spaces by platforms. This study begins to identify points of disjuncture between consumer desires and concerns, highlighting a current cultural difference between American acclimation to these devices and concerns within the Netherlands. No doubt this difference is connected to political, economic and social structures within these two countries, but overall our research reiterates a concern that users might grow "accustomed to and [begin] tolerating surveillance over time," specifically within the home (Lau, Zimmerman, and Schaub 2018: 3). Given device availability, this seems to be more the case in the US than in the Netherlands. Yet, even concern with potential broad-scale abuses of personal data (as seen, for example, in global responses to Facebook and Cambridge Analytica) may coexist with a desire for, acceptance of, or even resignation toward data gathering, such as collection through household IPAs. This latter perspective will likely not be evenly distributed within any given household as the decision to purchase, install, and ultimately welcome IPAs into a shared space may be the product of unequal power relations among co-habitants. The taken-forgranted presence of such devices in these households may further amplify such power discrepancies (e.g., its configuration and use by the most technologically literate member of a household).

It is clear that platforms' incursion into this context reinforces an ongoing acclimation to the ubiquitous presences of multiple types of sensors as they move from external locations (such as work and public spaces) to within the home itself. The connection of IPAs to other IoT devices in the home extends the knowledge that platforms can gain, particularly as most IoT devices are being made compatible with household IPAs though not manufactured by them. Taken together, this adds up to a notable extension of other ongoing infrastructural processes that Helmond (2015) has argued is occurring through the development of platforms: that is, for all their potential benefits and even with their concerns, household IPAs serve to make the home increasingly "platform ready." 


\section{References}

Brandt, Mathias. 2018. Wenig Echo in Deutschland [Not that much Echo in Germany]. Statista, February 13. https://de.statista.com/infografik/12884/smart-speaker-besitz-in-deutschland-und-den-usa/ [accessed January 7, 2019 ].

Braun, Virginia, and Victoria Clarke. 2006. Using Thematic Analysis in Psychology. Qualitative Research in Psychology 3 (2): $77-$ 101. https://doi.org/10.1191/1478088706qp063oa.

Clarke, Roger. 1988. Information Technology and Dataveillance. Communications of the ACM, 37 (5): 498-512.

Feldman, Russell. 2018. YouGov | Smart Speaker Ownership Doubles in Six Months. YouGov: What the World Thinks, 19 April. https://yougov.co.uk/topics/consumer/articles-reports/2018/04/19/smart-speaker-ownership-doubles-six-months [accessed January 7, 2019].

Guest, Greg, Kathleen M. MacQueen, and Emily E. Namey. 2011. Applied Thematic Analysis. Thousand Oaks, CA: SAGE.

Helmond, Anne. 2015. The Platformization of the Web: Making Web Data Platform Ready. Social Media + Society 1 (2): 1-11. https://doi.org/10.1177/2056305115603080.

Lau, Josephine, Benjamin Zimmerman, and Florian Schaub. 2018. Alexa, Are You Listening?: Privacy Perceptions, Concerns and Privacy-Seeking Behaviors with Smart Speakers. Proceedings of the ACM on Human-Computer Interaction 2 (CSCW): 131. https://doi.org/10.1145/3274371.

Lopatovska, Irene, Katrina Rink, Ian Knight, Kieran Raines, Kevin Cosenza, Harriet Williams, Perachya Sorsche, David Hirsch, Qi $\mathrm{Li}$, and Adrianna Martinez. Forthcoming. Talk to Me: Exploring User Interactions with the Amazon Alexa. Journal of Librarianship and Information Science. https://doi.org/10.1177/0961000618759414.

Luger, Ewa, and Abigail Sellen. 2016. "Like Having a Really Bad PA": The Gulf Between User Expectation and Experience of Conversational Agents. In Proceedings of the 2016 CHI Conference on Human Factors in Computing Systems, 5286-5297. New York: ACM. https://doi.org/10.1145/2858036.2858288.

Mols, Anouk, and Susanne Janssen. 2017. Not Interesting Enough to Be Followed by the NSA: An Analysis of Dutch Privacy Attitudes. Digital Journalism 5 (3): 277-98. https://doi.org/10.1080/21670811.2016.1234938.

Oudshoorn, Nelly E.J., and Trevor Pinch. 2003. How Users Matter: The Co-Construction of Users and Technologies. Cambridge, MA: MIT Press.

Perez, Sara. 2018. 47.3 Million US Adults Have Access to a Smart Speaker, Report Says. TechCrunch, 3 July. http://social.techcrunch.com/2018/03/07/47-3-million-u-s-adults-have-access-to-a-smart-speaker-report-says [accessed January 7, 2019].

Porcheron, Martin, Joel E. Fischer, and Sarah Sharples. 2017. "Do Animals Have Accents?": Talking with Agents in Multi-Party Conversation. In Proceedings of the 2017 ACM Conference on Computer Supported Cooperative Work and Social Computing, 207-219. CSCW'17. New York: ACM. https://doi.org/10.1145/2998181.2998298.

Ramaswamy, Sridhar. 2015. How Micro-Moments Are Changing the Rules. Think with Google, April. http://think.storage.googleapis.com/docs/how-micromoments-are-changing-rules.pdf [accessed January 7, 2019].

Sacks, Ethan. 2018. Alexa Privacy Fail Highlights Risks of Smart Speakers. NBC News, 26 May. https:/www.nbcnews.com/tech/innovation/alexa-privacy-fail-highlights-risks-smart-speakers-n877671 [accessed January 7, 2019].

Solove, Daniel J. 2011. Nothing to Hide: The False Tradeoff between Privacy and Security. New Haven, CT: Yale University Press.

Warren, Tom. 2018. Microsoft Is Now Selling Amazon's Echo Devices. The Verge, 17 November. https://www.theverge.com/2018/11/17/18099978/microsoft-store-amazon-echo-devices [accessed January 7, 2019].

Zeng, Eric, Shrirang Mare, and Franziska Roesner. 2017. End User Security and Privacy Concerns with Smart Homes. In Symposium on Usable Privacy and Security (SOUPS '17). https://www.usenix.org/conference/soups2017/technicalsessions/presentation/zeng [accessed January 7, 2019].

Zimmer, Michael, Priya Kumar, Jessica Vitak, Yuting Liao, and Katie Chamberlain Kritikos. Forthcoming. "There's Nothing Really They Can Do with This Information": Unpacking How Users Manage Privacy Boundaries for Personal Fitness Information. Information, Communication \& Society. https://doi.org/10.1080/1369118X.2018.1543442.

Zuboff, Shoshana. 2019. The Age of Surveillance Capitalism: The Fight for a Human Future at the New Frontier of Power. New York: Public Affairs. 